\title{
Thermal Quantum Coherence properties in Two-Qubit Heisenberg XXX Model in Non- homogeneous Magnetic Field
}

\author{
Asad $\mathrm{Ali}^{1}$, Muhammad Anees Khan ${ }^{2}$ \\ ${ }^{1}$ Quaid-i-Azam University, Islamabad 45320, Pakistan \\ ${ }^{2}$ Quaid-i-Azam University, Islamabad 45320, Pakistan \\ 1asadalinorth@gmail.com \\ 2aneesphyqau@gmail.com
}

\begin{abstract}
We investigate the behavior of thermal quantum coherence in the Heisenberg XXX model for twoqubit the system placed in independently controllable Inhomogeneous magnetic fields applied to two qubits respectively. We discuss the behavior of quantum coherence by systematically varying the coupling parameter, magnetic field, and temperature for both ferromagnetic and antiferromagnetic cases. The results show interesting behavior of quantum coherence in a certain range of parameters. Generally it is observed that quantum correlations decay with temperature, but in ferromagnetic case with uniform magnetic interaction, it rises with temperature up to a certain threshold value and ultimately it decreases its value to zero. Moreover, it is observed that preserving the quantum coherence for small temperatures is very hard with the increasing magnetic field because at small temperatures, quantum coherence decays sharply with the increase in magnetic field whereas at larger temperatures it decays completely at fairly large values of the magnetic field. The variation of quantum coherence with uniform magnetic field in antiferromagnetic case is observed to be Gaussian for larger temperature but at zero or nearly zero temperature it behaves as a constant function for uniform magnetic field up to a threshold value and then decays to zero with an infinite slope. This shows the signature of quantum phase transition from quantum nature to the classicality.
\end{abstract}

Keywords: Thermal quantum coherence. Heisenberg XXX model.

1

Introduction

Quantum coherence is a fundamental phenomenon in quantum physics and hence its quantification and detection is an elementary task. In the past, the distinction between quantum and classical coherence is made using phase-space distributions [1-2] and higher-order correlation functions [3]. While this gives some insight into the nature of coherence, these techniques don't quantify coherence in a rigorous sense. Recently a scheme for measuring coherence was developed by Baumgratz and co-workers in ref [4] based on the framework of quantum information theory. Rapid developments have been made in using it as a resource in quantum information theory [5-11]. Investigation on the role of quantum coherence in thermodynamic processes [12-14] assisted subspace discriminations [15], quantum state merging [16] and in the generation of Gaussian entanglement [17] has been carried out. Currently, there is a lot of interest in applying the procedure of quantifying quantum coherence and relating them to the experimental quantities infeasible systems like Bose-Einstein condensates [18-19] cavity opto-mechanical systems and spin systems [20-25].

In the context of quantum many-body systems, it was found in numerous works that entanglement can be used to detect quantum phase transitions in condensed matter systems [26-30]. This is 
natural since quantum correlations underlie both entanglement and quantum phase transitions. But entanglement accounts only for nonlocal quantum correlations and doesn't consider other kinds of correlations and quantum features [31-32]. To have a complete understanding of the role played by the quantumness of an object in physical phenomena it is important to consider the role played by other features like quantum coherence. In this paper, we study the behavior of thermal quantum coherence in two-qubit Heisenberg's XXX model under inhomogeneous magnetic field. Two cases, ferromagnetic and antiferromagnetic spin chain are discussed for two-qubit under uniform as well as nonuniform magnetic fields. We show that in the case of ferromagnetic two-qubit spin chain under uniform magnetic field, quantum coherence $C(\rho)$ increase with increase in temperature $T$ up to a certain threshold value scaled by the various fix values of magnetic field $B$ and $\mathrm{b}$ which control the degree of homogeneity in magnetic field.

The scheme of this paper runs as follows: In section 2 we discuss the two-qubit Heisenberg's XXX model under an inhomogeneous magnetic field. In section 3 , we define and discuss the recently proposed relative entropy-based definition of quantum coherence by [4]. In section 4, we calculate quantum coherence for our model and discuss its different cases with the help of obtained plots, and finally, in section 5 , we summarized the conclusions drawn.

\section{The Heisenberg XXX Model}

The Hamiltonian for two qubit system placed in magnetic field $(B-b)$ and $(B+b)$ to each qubit respectively is given by

$$
H_{X X X}=J\left(\sigma_{1}^{X} \sigma_{2}^{X}+\sigma_{1}^{Y} \sigma_{2}^{Y}+\sigma_{1}^{Z} \sigma_{2}^{Z}\right)+(B-b) \sigma_{1}^{Z}+(B+b) \sigma_{2}^{Z}
$$

Where $J$ is exchange coupling constant between spins in which $J<0$ corresponds to the ferromagnetic chain case and $J>0$ to the antiferromagnetic chain case, $\sigma_{n}^{\alpha}(\{\alpha=\{X, Y, Z\})$ is the Pauli spin operator. And $b$ indicates the degree of inhomogeneity of uniform magnetic field $B$. The two independently controllable magnetic fields are applied to two qubits respectively. Obviously the in-homogeneity of magnetic fields can be controlled by controlling the magnitude and direction. We notice that we are working in units so that $B, b$ and $J$ are dimensionless. We have also set Plank's constant and Boltzmann constant to be one $h=k=1$.

Using the standard computational basis $\{|00\rangle,|01\rangle,|10\rangle,|11\rangle\}$ The Hamiltonian (1) can be expressed under its matrix form as follows

$$
H=\left(\begin{array}{cccc}
J-2 B & 0 & 0 & 0 \\
0 & -J+2 b & 2 J & 0 \\
0 & 2 J & -j-2 b & 0 \\
0 & 0 & 0 & J+2 B
\end{array}\right)
$$

The eigenvectors and corresponding Eigen values of $H$ are given

$$
\begin{gathered}
E_{0}=-2 B+J, E_{1}=-J-\sqrt{J^{2}+b^{2}}, E_{2}=-J+\sqrt{J^{2}+b^{2}}, E_{3}=2 B+J \\
\psi_{0}=|00\rangle \\
\psi_{1}=N_{1}\left(a_{1}|01\rangle+|10\rangle\right) \\
\psi_{2}=N_{2}\left(a_{2}|01\rangle+|10\rangle\right) \\
\psi_{3}=|11\rangle
\end{gathered}
$$


Where $|0\rangle$ and $|1\rangle$ denote spin up and spin down states respectively

$$
a_{1}=\frac{-b-\sqrt{J^{2}+b^{2}}}{J}, a_{2}=\frac{-b+\sqrt{J^{2}+b^{2}}}{J}, N_{1}=\frac{1}{\sqrt{a_{1}^{2}+1}}, N_{2}=\frac{1}{\sqrt{a_{2}^{2}+1}}
$$

The expression for thermal equilibrium state of a quantum system is $\rho(T)=\frac{\exp \left(-\frac{H}{k T}\right)}{Z}$ and $Z=\operatorname{tr}\left[\exp \left(-\frac{H}{k T}\right)\right]$ is the partition function of the system and $\mathrm{k}$ is the Boltzmann constant. The partition function and the density matrix for the current system can be expressed as

$$
\begin{gathered}
Z=\exp \left(\frac{2 B-J}{k T}\right)+\exp \left(\frac{J+\sqrt{J^{2}+b^{2}}}{k T}\right)+\exp \left(\frac{J-\sqrt{J^{2}+b^{2}}}{k T}\right)+\exp \left(\frac{-2 B-J}{k T}\right) \\
\rho(T)=\frac{1}{Z}\left(\exp \left(\frac{2 B-J}{k T}\right)\left|\psi_{0}\right\rangle\left\langle\psi_{0}\left|+\exp \left(\frac{J+\sqrt{J^{2}+b^{2}}}{k T}\right)\right| \psi_{1}\right\rangle\left\langle\psi_{1}\left|+\exp \left(\frac{J-\sqrt{J^{2}+b^{2}}}{k T}\right)\right| \psi_{2}\right\rangle\left\langle\psi_{2}\right|+\right. \\
\left.\exp \left(\frac{-2 B-J}{k T}\right)\right)\left|\psi_{3}\right\rangle\left\langle\psi_{3}\right|
\end{gathered}
$$

Where,

$$
\rho(T)=\frac{1}{Z}\left(\begin{array}{cccc}
u & 0 & 0 & 0 \\
0 & w & y & 0 \\
0 & y & x & 0 \\
0 & 0 & 0 & v
\end{array}\right)
$$

$$
\begin{gathered}
u=\exp \left(-\frac{E_{0}}{k T}\right), \quad v=\exp \left(-\frac{E_{3}}{k T}\right) \\
y=N_{1}^{2} a_{1} \exp \left(-\frac{E_{1}}{k T}\right)+N_{2}^{2} a_{2} \exp \left(-\frac{E_{2}}{k T}\right) \\
w=N_{1}^{2} a_{1}^{2} \exp \left(-\frac{E_{1}}{k T}\right)+N_{2}^{2} a_{2}^{2} \exp \left(-\frac{E_{2}}{k T}\right) \\
x=N_{1}^{2} \exp \left(-\frac{E_{1}}{k T}\right)+N_{2}^{2} \exp \left(-\frac{E_{2}}{k T}\right)
\end{gathered}
$$

\section{Thermal quantum coherence}

We employ the relative entropy-based definition of quantum coherence in Heisenberg XXX model which is defined as follows.

For a quantum state, $|\psi\rangle$ the geometrical coherence can be witnessed by measuring $l_{1}$ trace norm between the state and the nearest incoherent state. Mathematically referring to fix basis $\{|i\rangle\}_{i=1}^{d}$

The $l_{1}$ norm of coherence is defined as [4] .

$$
C(\rho)_{l_{1}}=\sum_{i, j, i \neq j}\left|\rho_{i, j}\right|
$$

Where $\rho_{i, j}$ are the elements of density matrix $\rho\{=|\psi\rangle\langle\psi|\}$. Using von Neumann entropy $S(\rho)=$ $-\operatorname{tr}(\rho \log \rho)$, the relative entropy of coherence is defined as

$$
C(\rho)=S\left(\rho_{\text {diag }}\right)-S(\rho)
$$

Where $\rho_{\text {diag }}$ represents the diagonal state obtained from deleting all the off-diagonal elements of $\rho$.

\section{$4 \quad$ Numerical calculation and theoretical results}


We initially consider the case of ferromagnetic chain and then antiferromagnetic one. In them, there are further two magnetic field interactions cases; uniform magnetic field ( $b=0$ and $B \neq 0)$ and nonuniform magnetic field ( $B=0$ and $b \neq 0$ ).

When $J<0$ : For ferromagnetic case we set $J=-1$ for the convenience of discussion. In this case, there are further two case; uniform magnetic field $(b=0, B \neq 0)$ and non uniform magnetic field $(B=0, b \neq 0)$.

\subsection{Ferromagnetic case with uniform magnetic field}

The behavior of quantum coherence $C(\rho)$ under uniform magnetic field as a function of magnetic field $B$ and temperature $T$ when $b=0$ is shown below.

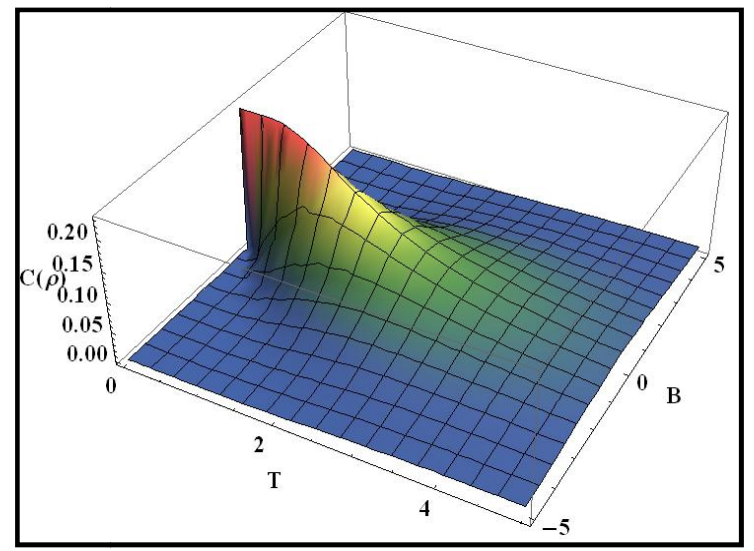

Figure 1. Quantum coherence versus $C(\rho)$ uniform magnetic field $B$ and Temperature $T$. The 2D projections of this 3D plots reveal some interesting results at different values of certain parameters as shown below.

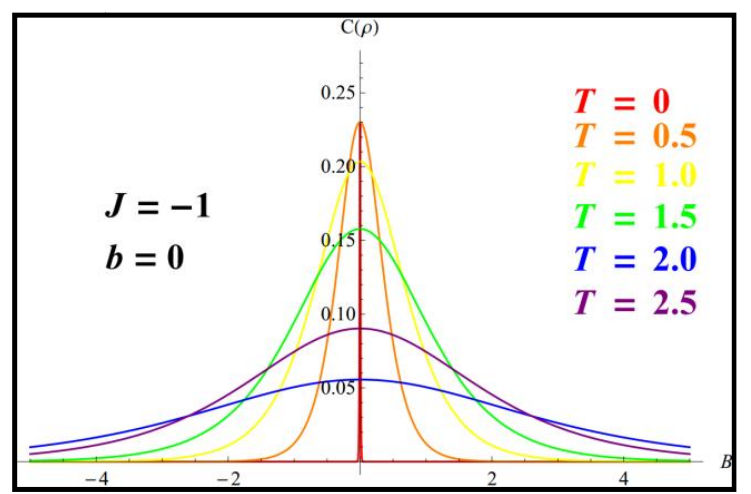

Figure 2.Quantum coherence $C(\rho)$ versus uniform magnetic field $B$ for certain fix values of temperature.

From the figure, we can know that the thermal quantum coherence is symmetric with respect to the uniform magnetic field for different values of temperatures. It furthermore shows the delicacy in the decay rate of coherence with increasing values of the magnetic field at different fixed temperature values. As we increase the magnetic field, quantum coherence decays rapidly as temperature is small and slowly when temperature increases slightly. 


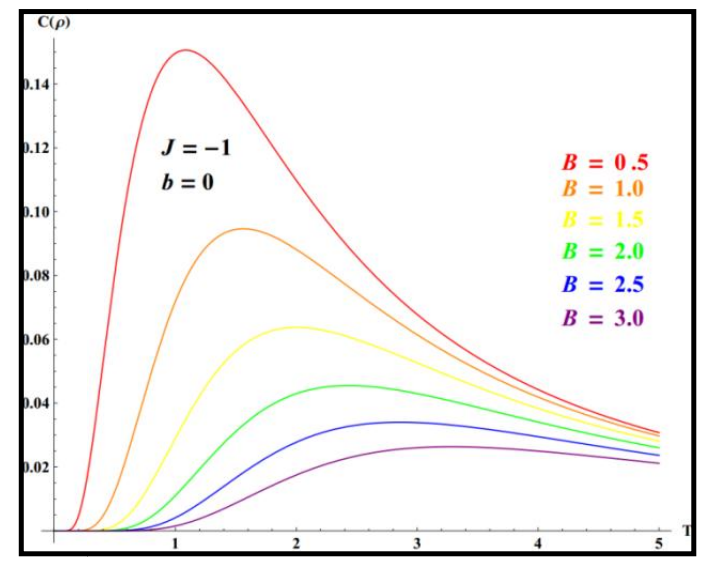

Figure 3. $C(\rho)$ Versus $T$ for different fix values of $B$.

It is seen from figure 3 that for low temperature range from 0 to around 2, the amount of quantum coherence improves with increase in temperature for fixed values of magnetic fields. For small magnetic field quantum coherence increase to maximum rapidly and decays slowly whereas for large magnetic field values, quantum coherence increase relatively slowly.

\subsection{Ferromagnetic case with non-uniform magnetic field}

The behavior of quantum coherence $C(\rho)$ under nonuniform magnetic field as a function of magnetic field $b$ and temperature $T$ when $\mathrm{B}=0$ is shown below.

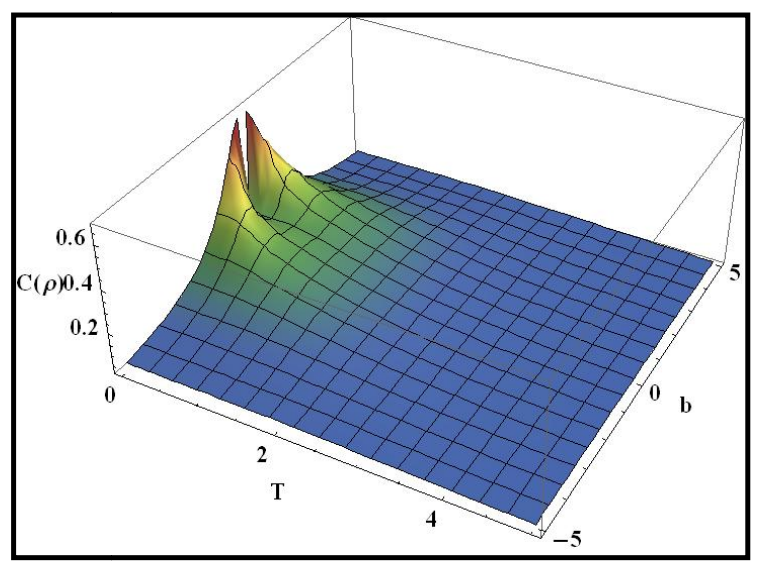

Figure 4.Dependence of $C(\rho)$ with $T$ and $b$ for $B=0$.The 2D projections of this 3D plots reveals some interesting results at different values of certain parameters as shown below. 


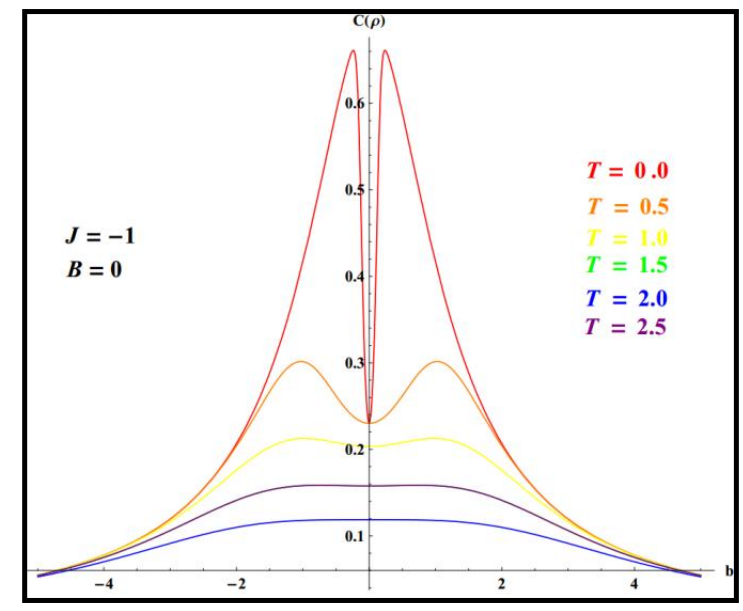

Figure 5.Dependence of $C(\rho)$ on nonuniform magnetic field $b$ for various temperatures.

In figure 5, we display the quantum coherence behavior for ferromagnetic case at different temperatures as a function of nonuniform magnetic field $b$. From the figure, we know that quantum correlations are symmetric with respect to the nonuniform magnetic field $b$. It furthermore shows the interesting dynamic of quantum coherence as a function of $b$. At different fix values of temperatures, the quantum coherence initially increases with nonuniform magnetic field up to a certain threshold value and then decays to zero. This interesting behavior is very prominent at low temperatures as compare to larger temperatures.

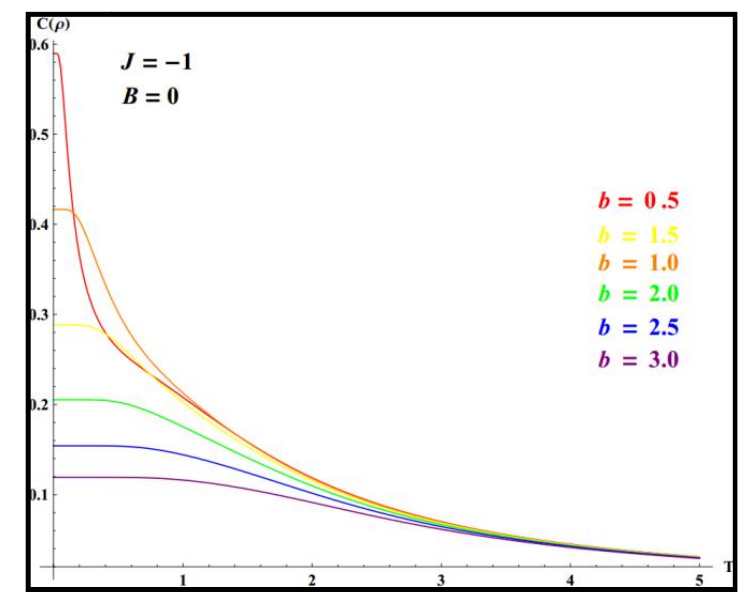

Figure 6. Thermal quantum coherence as a function of temperature for various values of the nonuniform magnetic field.

From the figure, it can be seen that quantum coherence decreases with the temperature at various values of the nonuniform magnetic field parameters.

When $J>0$ : For antiferromagnetic case, we set $L=+1$ for the convenience of discussion. There are further two cases; uniform magnetic field $(b=0$ and $B \neq 0)$ and non uniform magnetic field $(B=0$ and $b \neq 0$ ).

\subsection{Antiferromagnetic case with uniform magnetic field}

The behavior of quantum coherence $C(\rho)$ under uniform magnetic field as a function of magnetic field $B$ and temperature $T$ when $b=0$ is shown below. 


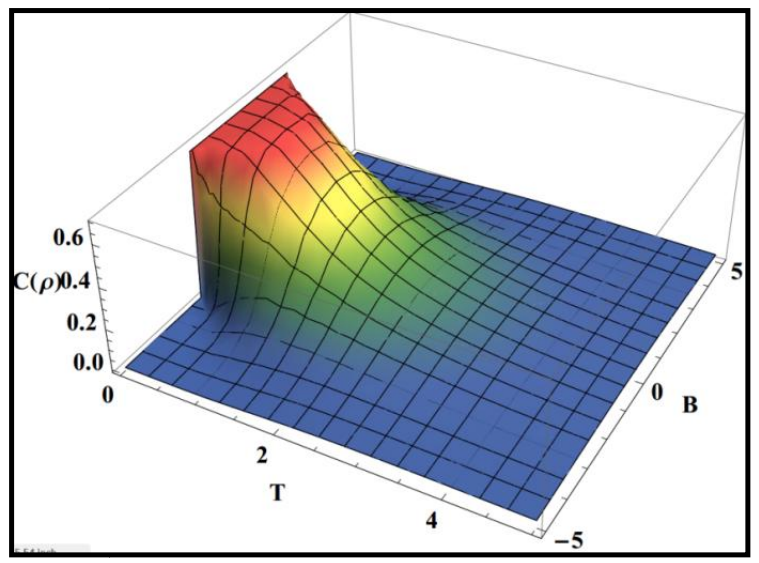

Figure 7. Quantum coherence $C(\rho)$ versus temperature and uniform magnetic field $B(J=+1$ and $b=0$ ).

The $2 \mathrm{D}$ projections of this $3 \mathrm{D}$ plots reveals some interesting results at different values of certain parameters as shown below.

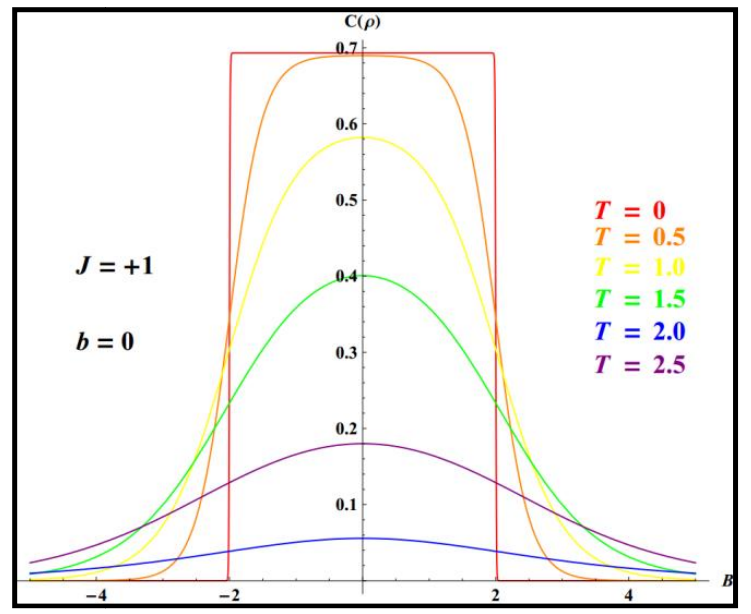

Figure 8. Quantum coherence $C(\rho)$ versus $B$ at different fix values of T for $b=0$.

From the figure, we can know that the thermal quantum coherence is symmetric with respect to the uniform magnetic field for different values of temperatures. Furthermore, it shows the decrease in the amount of coherence with increasing value of the magnetic field at different temperatures. For larger temperature values the graph of quantum coherence versus uniform magnetic field behaves like Gaussian function but for small values of temperature (say at $T=0.5$ and $T=0$ ), It behaves like a rectangular function. In fact at $T=0$, quantum coherence becomes a constant function of $B$ from $-2<B<2$ and beyond that at $B= \pm 2$ it goes to zero with an infinite slope which is very interesting. This shows that at zero temperature (nearly zero temperature as well), the quantum coherence remains maximum under a certain range of magnetic field (maximum quantumness) and then it becomes clearly zero (no quantumness) within no time. This is a clear signature of sudden phase transition which shows the emergence of classicality from quantumness. However, this phase transition becomes faint at larger temperatures. 


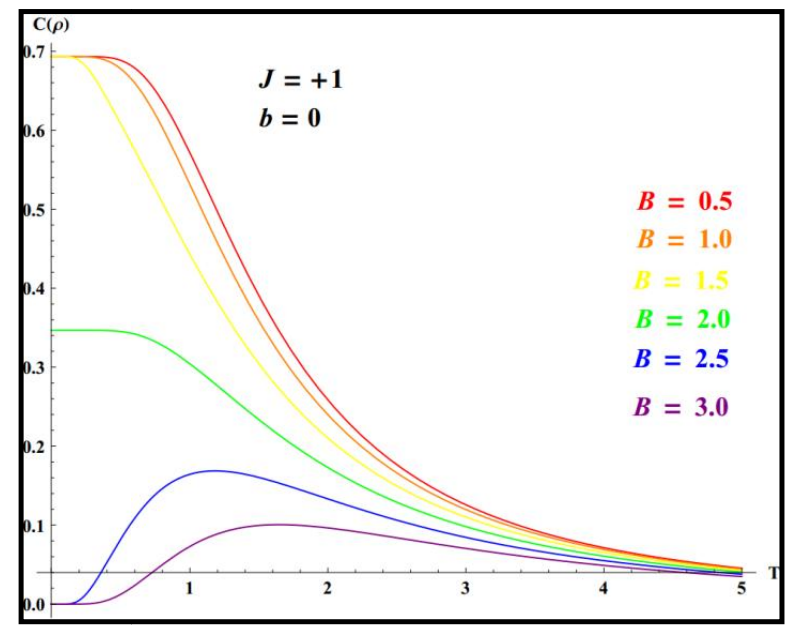

Figure 9. Quantum coherence $C(\rho)$ versus Temperature for various values of uniform magnetic field $B(J=+1$ and $b=0)$. It can be seen that in this nonuniform ferromagnetic case quantum coherence decreases with the increase in the values of both the magnetic field as well as temperature.

\section{Antiferromagnetic case with non-uniform magnetic field}

The behavior of quantum coherence $C(\rho)$ in the case of non uniform antiferromagnetic magnetic field $(b \neq 0$ and $B=0)$ as a function of magnetic field $B$ and temperature $T$ when $b=0$ is shown below

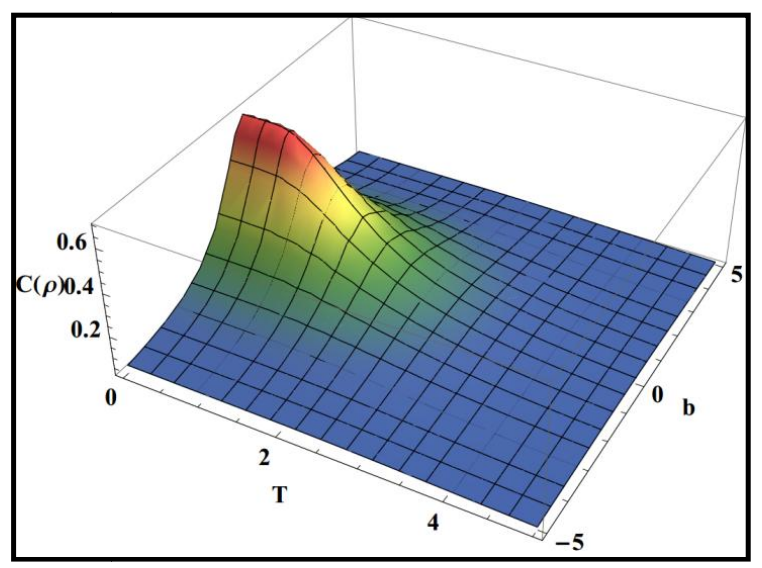

Figure 10. Quantum coherence $C(\rho)$ versus temperature T and non uniform magnetic field b

The corresponding 2D projections of this 3D plot at different values of certain parameters as shown below. 


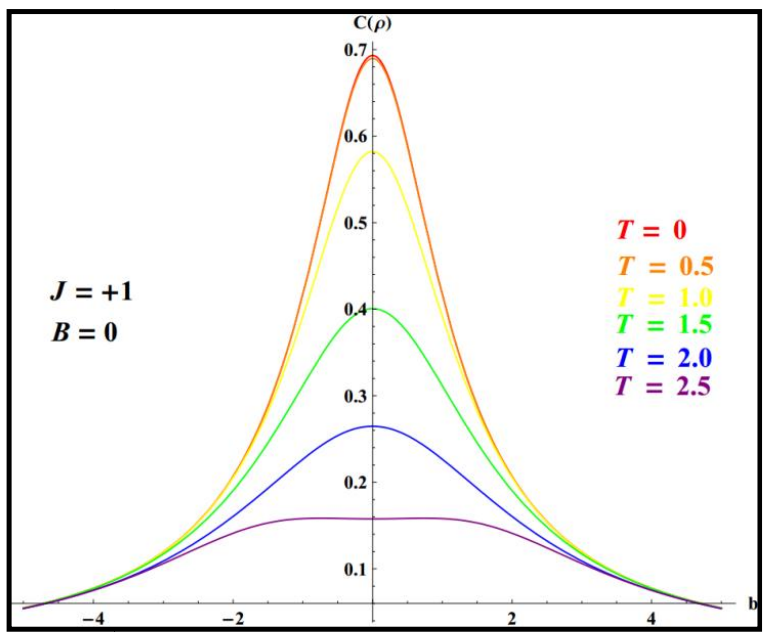

Figure 11. Quantum coherence $C(\rho)$ versus nonuniform magnetic field $\mathrm{b}$ at various temperatures $\mathrm{T}$

Figure show a decrease in the amount of quantum coherence with the increase in values of both temperature as well as nonuniform magnetic field parameter.

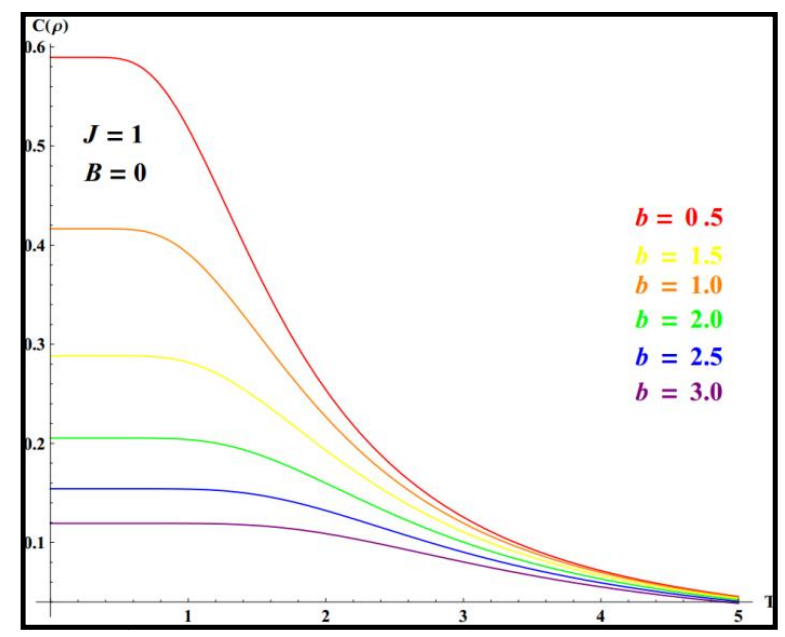

Figure 12.Quantum coherence $C(\rho)$ versus temperature at various values of nonuniform magnetic field. It can be seen that the quantum coherence decays with time for different values of nonniform magnetic field parameters.

\section{Conclusion}

The quantum coherence is a key characteristic feature of quantum theory and it is proved to be a resource in various quantum information and estimation protocols and is primarily accountable for the advantage offered by quantum tasks versus classical ones. While the theory of quantum coherence is well established historically in quantum optics but a rigorous framework for its explicit quantification for generals states in information-theoretic terms has been attempted in the last five years.

Quantum coherence like any other quantum property which is based on the quantum superposition principle within a system as well as among multipartite systems shows fragility toward environmental noise like thermal agitations and perturbations. One may ask how thermal fluctuation and perturbations affect quantum coherence in many-body quantum systems. This is the question that we asked and investigated in this paper for two-qubit Heisenberg XXX models under 
inhomogeneous magnetic field perturbation and thermal agitation of an environmental bath. We have tried to extract the information from the given model by discussing each and every possible case by systematically varying the values and variables.

We discussed ferromagnetic and antiferromagnetic spin chain cases under uniform and nonuniform magnetic field perturbations placed in an environmental bath. In particular, we have shown how different factors like exchange coupling constant and magnetic fields interplay with temperature to preserve or collapse the quantum coherence. The interesting behavior of quantum coherence with uniform magnetic field in antiferromagnetic case is observed to be Gaussian for larger temperature but at zero or nearly zero temperature it behaves as a constant function for uniform magnetic field up to a threshold value and then decays to zero with an infinite slope. This shows the signature of quantum phase transition from quantum nature to the classicality.

\section{References}

[1]Glauber, R.J., 1963. Coherent and incoherent states of the radiation field. Physical Review, 131(6), p.2766.

[2] Sudarshan, E.C.G., 1963. Equivalence of semiclassical and quantum mechanical descriptions of statistical light beams. Physical Review Letters, 10(7), p.277.

[3] Scully, M.O. and Zubairy, M.S., 1999. Quantum optics.

[4]Baumgratz, T., Cramer, M. and Plenio, M.B., 2014. Quantifying coherence. Physical review letters, 113(14), p.140401.

[5] Streltsov, A., Adesso, G. and Plenio, M.B., 2017. Colloquium: Quantum coherence as a resource. Reviews of Modern Physics, 89(4), p.041003.

[6] Marvian, I. and Spekkens, R.W., 2016. How to quantify coherence: Distinguishing speakable and unspeakable notions. Physical Review A, 94(5), p.052324.

[7]Winter, A. and Yang, D., 2016. Operational resource theory of coherence. Physical review letters, 116(12), p.120404.

[8]Brandao, F.G. and Gour, G., 2015. Reversible framework for quantum resource theories. Physical review letters, 115(7), p.070503.

[9]Brandao, F.G. and Gour, G., 2015. Reversible framework for quantum resource theories. Physical review letters, 115(7), p.070503.

[10]Chitambar, E. and Hsieh, M.H., 2016. Relating the resource theories of entanglement and quantum coherence. Physical review letters, 117(2), p.020402.

[11]Del Rio, L., Kraemer, L. and Renner, R., 2015. Resource theories of knowledge. arXiv preprint arXiv:1511.08818.

[12] Lostaglio, M., Jennings, D. and Rudolph, T., 2015. Description of quantum coherence in thermodynamic processes requires constraints beyond free energy. Nature communications, 6(1), pp.1-9.

[13]Narasimhachar, V. and Gour, G., 2015. Low-temperature thermodynamics with quantum coherence. Nature communications, 6(1), pp.1-6.

[14]Lostaglio, M., Korzekwa, K., Jennings, D. and Rudolph, T., 2015. Quantum coherence, timetranslation symmetry, and thermodynamics. Physical review X, 5(2), p.021001.

[15]Zhang, F.L. and Wang, T., 2017. Intrinsic coherence in assisted sub-state discrimination. EPL (Europhysics Letters), 117(1), p.10013. 
[16]Streltsov, A., Chitambar, E., Rana, S., Bera, M.N., Winter, A. and Lewenstein, M., 2016. Entanglement and coherence in quantum state merging. Physical review letters, 116(24), p.240405.

[17] Wang, Z.X., Wang, S., Ma, T., Wang, T.J. and Wang, C., 2016. Gaussian entanglement generation from coherence using beam-splitters. Scientific reports, 6(1), pp.1-7.

[18]Opanchuk, B., Rosales-Zárate, L., Teh, R.Y. and Reid, M.D., 2016. Quantifying the mesoscopic quantum coherence of approximate noon states and spin-squeezed two-mode bose-einstein condensates. Physical Review A, 94(6), p.062125.

[19]Pyrkov, A.N. and Byrnes, T., 2014. Full-Bloch-sphere teleportation of spinor Bose-Einstein condensates and spin ensembles. Physical Review A, 90(6), p.062336.

[20]Karpat, G., Çakmak, B. and Fanchini, F.F., 2014. Quantum coherence and uncertainty in the anisotropic XY chain. Physical Review B, 90(10), p.104431.

[21]Malvezzi, A.L., Karpat, 1.G., Çakmak, B., Fanchini, F.F., Debarba, T. and Vianna, R.O., 2016. Quantum correlations and coherence in spin-1 Heisenberg chains. Physical Review B, 93(18), p.184428.

[22]Chen, J.J., Cui, J., Zhang, Y.R. and Fan, H., 2016. Coherence susceptibility as a probe of quantum phase transitions. Physical Review A, 94(2), p.022112.

[23]Li, Y.C. and Lin, H.Q., 2016. Quantum coherence and quantum phase transitions. Scientific reports, 6(1), pp.1-8.

[24]Cheng, W., Zhang, Z., Gong, L. and Zhao, S., 2016. Finite-temperature scaling of quantum coherence near criticality in a spin chain. The European Physical Journal B, 89(6), pp.1-6.

[25]Çakmak, B., Karpat, G. and Fanchini, F.F., 2015. Factorization and criticality in the anisotropic xy chain via correlations. Entropy, 17(2), pp.790-817.

[26] Osterloh, A., Amico, L., Falci, G. and Fazio, R., 2002. Scaling of entanglement close to a quantum phase transition. Nature, 416(6881), pp.608-610.

[27]Osborne, T.J. and Nielsen, M.A., 2002. Entanglement in a simple quantum phase transition. Physical Review A, 66(3), p.032110.

[28]Vidal, G., Latorre, J.I., Rico, E. and Kitaev, A., 2003. Entanglement in quantum critical phenomena. Physical review letters, 90(22), p.227902.

[29]Wu, L.A., Sarandy, M.S. and Lidar, D.A., 2004. Quantum phase transitions and bipartite entanglement. Physical review letters, 93(25), p.250404.

[30]Mehran, E., Mahdavifar, S. and Jafari, R., 2014. Induced effects of the Dzyaloshinskii-Moriya interaction on the thermal entanglement in spin-1/2 Heisenberg chains. Physical Review A, 89(4), p.042306.

[31]Henderson, L. and Vedral, V., 2001. Classical, quantum and total correlations. Journal of physics A: mathematical and general, 34(35), p.6899.

[32]Ollivier, H. and Zurek, W.H., 2001. Quantum discord: a measure of the quantumness of correlations. Physical review letters, 88(1), p.017901. 